TRANSACTIONS OF THE

AMERICAN MATHEMATICAL SOCIETY

Volume 352, Number 6, Pages 2701-2721

S $0002-9947(00) 02581-2$

Article electronically published on February 28, 2000

\title{
PROBLÈME DE DIRICHLET POUR UNE ÉQUATION DE MONGE-AMPÈRE RÉELLE ELLIPTIQUE DÉGÉNÉRÉE EN DIMENSION $n$
}

\author{
AMEL ATALLAH
}

RÉsumé. On considère dans un ouvert borné $\Omega$ de $\mathbb{R}^{n}$, à bord régulier, le problème de Dirichlet

$$
\left\{\begin{array}{l}
\operatorname{det} u_{i j}=f(x) \text { dans } \Omega \\
\left.u\right|_{\partial \Omega}=\varphi
\end{array}\right.
$$

où $f \in C^{s_{*}}(\bar{\Omega}), \varphi \in C^{s_{*}+2, \alpha}(\Omega), f$ est positive et s'annule sur $\Sigma$ un ensemble fini de points de $\Omega$. On démontre alors sous certaines hypothèses sur $\varphi$ et si $\left|\operatorname{det} \varphi_{i j}-f\right|_{C^{s_{*}}}$ est assez petit, que le problème (1) possède une solution convexe unique $u \in C^{\left[s_{*}-3-n / 2\right]}(\bar{\Omega})$.

Abstract. We consider in a bounded open set $\Omega$ of $\mathbb{R}^{n}$, with regular boundary, the Dirichlet problem

$$
\left\{\begin{array}{l}
\operatorname{det} u_{i j}=f(x) \text { in } \Omega, \\
\left.u\right|_{\partial \Omega}=\varphi,
\end{array}\right.
$$

where $f \in C^{s_{*}}(\bar{\Omega}), \varphi \in C^{s_{*}+2, \alpha}(\Omega), f$ is positive and vanishes on $\Sigma$, a finite set of points in $\Omega$. We prove, under some hypothesis on $\varphi$ and if $\left|\operatorname{det} \varphi_{i j}-f\right|_{C^{s_{*}}}$ is sufficiently small, that the problem (1) has a unique convex solution $u \in$ $C^{\left[s_{*}-3-n / 2\right]}(\bar{\Omega})$.

\section{INTRODUCTION}

Plusieurs travaux ont été consacrés à l'étude de l'existence de solutions convexes régulières pour l'équation de Monge-Ampère

$$
\operatorname{det} u_{i j}=f(x, u, \nabla u)
$$

dans un domaine borné strictement convexe $\Omega$ de $\mathbb{R}^{n}$.

Dans le cas où (0.1) est elliptique, c'est-à-dire $f$ est strictement positive dans $\bar{\Omega}$, Caffarelli, Nirenberg, Spruck C.N.S.1, on montré que si $f$ est $C^{\infty}$ et $f_{u} \geq 0$, le problème de Dirichlet associé à (0.1) possède une solution unique strictement convexe $u \in C^{\infty}(\bar{\Omega})$, sous l'hypothèse d'existence d'une sous-solution. On cite aussi les travaux de [C.N.S.2], [E], [K], [T], [U]...

Dans le cas où $f$ est positive ou nulle, Lin $[\mathrm{L}]$ en dimension $n=2$, a montré l'existence d'une solution locale de classe $C^{s}$; puis Hong et Zuily [H.Z] en dimension

Received by the editors April 17, 1995.

1991 Mathematics Subject Classification. Primary 35J25, 35J70, 35Q99.

Key words and phrases. Equation de Monge-Ampère, problème de Dirichlet, équation elliptique dégénérée. 
$n$ quelconque ont montré, si $f$ s'annule à un ordre fini, l'existence d'une solution locale convexe de classe $C^{\infty}$.

Concernant le problème de Dirichlet associé à l'équation (0.1) dans le cas où $f$ peut s'annuler, l'existence d'une solution globale assez régulière n'est pas toujours possible pour des données quelconques, et dans le cas général la solution est au maximum de classe $C^{1,1}$ dans $\bar{\Omega}$. On peut citer l'exemple dû à Urbas [U] dans le cas où $f$ est identiquement nulle, également ceux d'Amano [A] et Zuily [Z] dans des cas un peu plus généraux.

Signalons aussi les travaux de C.N.S.3 qui montrent dans le cas où $f$ est identiquement nulle, si $\partial \Omega$ et $\varphi$ sont de classe $C^{3,1}$, que la solution est de classe $C^{1,1}$ dans $\bar{\Omega}$. Ils montrent aussi que ce résultat est optimal en donnant un exemple où $\varphi \in C^{3,1-2 \varepsilon}(\partial \Omega), \varepsilon$ positif assez petit et la solution $u \in C^{1,1-\varepsilon}(\bar{\Omega})$ mais $u \notin C^{1,1}(\Omega)$. La difficulté provient du fait que $f$ pouvant s'annuler, le linéarisé a un symbole positif ou nul et on ne peut donc pas appliquer les méthodes usuelles pour les opérateurs elliptiques.

L'objet de ce travail est de traiter un cas particulier où $f$ peut s'annuler. Plus précisément on considère dans un ouvert borné $\Omega$ de $\mathbb{R}^{n}$ à bord $C^{\infty}$ le problème de Dirichlet

$$
\left\{\begin{array}{l}
\operatorname{det} u_{i j}=f(x) \operatorname{dans} \Omega, \\
\left.u\right|_{\partial \Omega}=\varphi
\end{array}\right.
$$

où $f$ et $\varphi$ sont assez régulières et $\left(u_{i j}\right)$ est la matrice Henssienne de $u$. On démontre alors dans le cas où $f$ s'annule en un nombre fini de points à l'intérieur de $\Omega$, sous certaines hypothèses sur $\varphi$, et si $\left|\operatorname{det} \varphi_{i j}-f\right|_{s_{*}}$ est assez petit, l'existence d'une solution convexe unique assez régulière jusqu'au bord pour le problème (0.2). La preuve consiste à établir des estimations a priori pour des opérateurs elliptiques dégénérés puis à construire pour le linéarisé un schéma itératif approprié du type Nash-Moser dont la convergence assure l'existence de la solution. Ce résultat généralise à la dimension $n$ quelconque les travaux d'Amano $[\mathrm{A}]$ qui démontre un résultat analogue en dimension $n=2$.

\section{Notations ET RÉsultats}

On considère dans un ouvert borné $\Omega$ de $\mathbb{R}^{n}$ à bord $C^{\infty}$, le problème de Dirichlet

$$
\operatorname{det} u_{i j}=f(x) \operatorname{dans} \Omega,\left.\quad u\right|_{\partial \Omega}=\varphi .
$$

Ici $u$ est une fonction réele, $u_{i j}=\frac{\partial^{2} u}{\partial x_{i} \partial x_{j}}$ et $\varphi$ est une fonction définie sur $\bar{\Omega}$.

Soit $\Sigma$ un ensemble fini de points de $\Omega$, on supposera dans ce qui suit

$$
\left\{\begin{array}{l}
\text { (i) } f \geq 0 \text { dans } \bar{\Omega}, \\
\text { (ii) } f^{-1}(0)=\Sigma
\end{array}\right.
$$

et

$$
\left\{\begin{array}{l}
\text { (i) }\left.\quad\left(\varphi_{i j}\right)\right|_{\bar{\Omega} \backslash \Sigma} \text { est strictement convexe, }\left.\left(\varphi_{i j}\right)\right|_{\Sigma} \text { est de rang }(n-1), \\
\text { (ii) les valeurs propres de }\left(\varphi_{i j}\right) \text { sur } \Sigma \text { sont distinctes. }
\end{array}\right.
$$

On a alors le résultat suivant: 
Théorème A. Pour tout entier $s_{*} \geq 7+n$, tout $\left.\alpha \in\right] 0,1[$ et toute fonction $\varphi \in C^{s_{*}+2, \alpha}(\bar{\Omega})$ vérifiant (I.3), il existe une constante $\varepsilon_{0}>0$ telle que pour toute fonction $f \in C^{s_{*}}(\bar{\Omega})$ satisfaisant (I.2) et

$$
\left|\operatorname{det} \varphi_{i j}-f\right|_{C^{s_{*}}} \leq \varepsilon_{0}
$$

le problème (1.1) possède une solution convexe unique u dans $C^{\left[s_{*}-3-n / 2\right]}(\bar{\Omega})$.

Remarque. Dans le cas où $\Sigma=\left\{x_{0}\right\}$, on pourra considérer par exemple pour $\varepsilon$ assez petit, la fonction $f$ définie par $f(x)=\operatorname{det} \varphi_{i j}+\varepsilon\left|x-x_{0}\right|^{2}$.

Les normes que nous utiliserons sont notées comme suit

$$
||_{k}=\|\|_{C^{k}(\bar{\Omega})},\|\|_{k}=\|\|_{H^{k}(\Omega)} \text { et }||_{k, \alpha}=\|\|_{C^{k, \alpha}(\bar{\Omega})}
$$

où $k \in \mathbb{N}$ et $\alpha \in] 0,1[$.

\section{Preuve Du ThÉorème A}

\section{II.1. Préliminaires.}

A. On effectue dans ce paragraphe une réduction du problème et on démontre quelques résultats techniques qui nous seront utiles dans la suite. Tout d'abord on pourra supposer que $\Sigma$ est réduit à un point que l'on peut supposer être l'origine. De plus comme l'équation (I.1) est invariante par rotation de coordonnées et d'après (I.3) on pourra supposer qu'il existe des coordonnées dans lesquelles on peut écrire:

$$
\varphi_{i j}(0)=\sigma_{i} \delta_{i j}, \quad i, j=1, \ldots, n,
$$

où $\sigma_{i}>0$ pour $i=1, \ldots, n-1, \sigma_{n}=0$ et $\sigma_{i} \neq \sigma_{j}$ pour $i \neq j$.

Pour $\varepsilon \geq 0, w \in C^{2}(\bar{\Omega})$ on pose

$$
\left\{\begin{array}{l}
\Phi_{i j}(x, \varepsilon, w)=\varphi_{i j}+\varepsilon w_{i j}, \quad 1 \leq i, j \leq n \\
\Phi=\left(\Phi_{i j}(x, \varepsilon, w)\right)
\end{array}\right.
$$

Notons que $\Phi^{i j}=\frac{\partial F}{\partial \Phi_{i j}}(\Phi)$, où $F$ est la fonction déterminant. On a alors le résultat suivant:

Lemme II.1. Pour $\varepsilon \geq 0, w \in C^{2}(\bar{\Omega})$ et $x \in \bar{\Omega}$, il existe une matrice orthogonale $T=T(x, \varepsilon, w)$ telle que

$$
\begin{gathered}
T \Phi^{t} T=\operatorname{diag}\left(\lambda_{1}, \ldots, \lambda_{n}\right), \quad T \widetilde{\Phi}^{t} T=\operatorname{diag}\left(\tilde{\lambda}_{1}, \ldots, \tilde{\lambda}_{n}\right), \\
\tilde{\lambda}_{i}=\prod_{j \neq i} \lambda_{j}, \quad i=1, \ldots, n .
\end{gathered}
$$

Soit $\kappa \in] 0,1\left[, \kappa \leq \frac{\alpha}{4}\right.$ où $\alpha$ est tel que $\varphi \in C^{s_{*}+2, \alpha}(\bar{\Omega})$.

Le résultat suivant précise la nature des valeurs propres de $\Phi$.

Lemme II.2. Il existe des constantes $\varepsilon_{1}>0, \delta_{1}>0$ et $M>0$ qui ne dépendent que de $\varphi, n, \Omega$ telles que, en posant $V=\left\{|x| \leq \delta_{1}, 0 \leq \varepsilon \leq \varepsilon_{1}, w \in C^{3, \kappa}(\bar{\Omega}),|w|_{3, \kappa} \leq 1\right\}$ on ait

(i) les valeurs propres $\lambda_{i}, i=1, \ldots, n$, det $\Phi$ sont distinctes dans $V$ et de classe $C^{1}$ dans $\stackrel{\circ}{V}$. De plus $\lambda_{i}>0$ dans $V$ pour $i=1, \ldots, n-1$. 
(ii) $\operatorname{Pour}(x, \varepsilon, w) \in V$ :

$$
\sum_{i=1}^{n}\left|\sigma_{i}-\lambda_{i}(x, \varepsilon, w)\right|+\left|\Phi^{n n}(x, \varepsilon, w)-\prod_{i=1}^{n-1} \sigma_{i}\right| \leq M(\varepsilon+|x|),
$$

(iii) $\operatorname{pour}(x, \varepsilon, w) \in V$ et $i=1, \ldots, n-1$

$$
\lambda_{i} \geq \inf _{1 \leq i \leq n-1} \sigma_{i}-M \delta_{1}-(M+1) \varepsilon_{1}>0 \text { et } \Phi^{n n} \geq \prod_{i=1}^{n-1} \sigma_{i}-M \delta_{1}-M \varepsilon_{1}>0 .
$$

Preuve. Pour $(x, \varepsilon, w, \lambda) \in \bar{\Omega} \times \mathbb{R}_{+} \times C^{3}(\bar{\Omega}) \times \mathbb{R}$ on définit

$$
g(x, \varepsilon, w, \lambda)=\operatorname{det}\left(\varphi_{i j}+\varepsilon w_{i j}-\lambda \delta_{i j}\right) .
$$

$g$ est une fonction de classe $C^{1}$ de ses arguments. Soit $i \in\{1, \ldots, n\}$; d'après (II.1) on a

$$
g\left(0,0,0, \sigma_{i}\right)=0 \text { et } \frac{\partial g}{\partial \lambda}\left(0,0,0, \sigma_{i}\right) \neq 0 .
$$

Le théorème des fonctions implicites implique qu'il existe des constantes $\varepsilon_{1}>$ $0, \delta_{1}>0$ et $M>0$ telles que l'on ait (i) et (iii). De plus d'après (II.1) on a:

$$
\frac{\partial F}{\partial u_{n n}}\left(\varphi_{i j}\right)(0)=\Phi^{n n}(0,0, w)=\prod_{i=1}^{n-1} \sigma_{i}>0
$$

d'où (iii).

Voici le résultat essentiel de ce paragraphe. Posons pour $\varepsilon>0, x \in \bar{\Omega}$ et $w \in$ $C^{2}(\bar{\Omega})$

$$
G(w)=\frac{1}{\varepsilon}\left[F\left(\Phi_{i j}(x, \varepsilon, w)\right)-f\right] .
$$

Le linéarisé de $G$ en $w$ est

$$
L_{G}(w)=\sum_{i, j=1}^{n} \Phi^{i j} \partial_{i} \partial_{j}
$$

On a alors

Proposition II.3. Il existe une constante positive $\varepsilon_{2}$ telle que pour $0<\varepsilon \leq \varepsilon_{2}$, $w \in C^{3, \kappa}(\bar{\Omega}),|w|_{3, \kappa} \leq 1$, l'opérateur

$$
L=-L_{G}(w)-\theta \Delta, \text { oѝ } \theta=\sup _{x \in \bar{\Omega}}|G(w)|,
$$

un a symbole positif ou nul sur $\bar{\Omega} \times \mathbb{R}^{n}$. (Ici $\Delta=\sum_{i=1}^{n} \frac{\partial^{2}}{\partial x_{i}^{2}}$.)

Preuve. Le symbole de $L$ est

$$
A=\theta|\xi|^{2}+\sum_{i, j=1}^{n} \Phi^{i j} \xi_{i} \xi_{j}
$$

Tout d'abord montrons que $A \geq 0$ pour $|x| \leq \delta_{1}$ et $\xi \in \mathbb{R}^{n}$. Posons $\xi={ }^{t} T \tilde{\xi}$ où $T$ est définie en (II.4). D'après (II.10) on a

$$
A=\theta|\tilde{\xi}|^{2}+\left\langle T \widetilde{\Phi}^{t} T \tilde{\xi}, \tilde{\xi}\right\rangle .
$$


En utilisant (II.4) et (II.5) on obtient

$$
A=\theta|\tilde{\xi}|^{2}+\sum_{i=1}^{n} \tilde{\lambda}_{i} \tilde{\xi}_{i}^{2} \text { où } \tilde{\lambda}_{i}=\prod_{\substack{j=1 \\ j \neq i}}^{n} \lambda_{j}
$$

D'après (II.7) et (II.8) on a:

$$
\begin{aligned}
A & =\left(\theta+\prod_{i=1}^{n-1} \lambda_{i}\right) \tilde{\xi}_{n}^{2}+\sum_{i=1}^{n-1}\left(\frac{\operatorname{det} \Phi}{\lambda_{i}}+\theta\right) \tilde{\xi}_{i}^{2} \\
& =\left(\theta+\prod_{i=1}^{n-1} \lambda_{i}\right) \tilde{\xi}_{n}^{2}+\sum_{i=1}^{n-1} \frac{\varepsilon G+f+\theta \lambda_{i}}{\lambda_{i}} \tilde{\xi}_{i}^{2}
\end{aligned}
$$

(II.7) implique pour $i=1, \ldots, n-1,|x| \leq \delta_{1}, \varepsilon \leq \varepsilon_{1},|w|_{3, \kappa} \leq 1$

$$
\theta \lambda_{i}+\varepsilon G \geq \theta\left(\sigma_{i}-M \delta_{1}-(M+1) \varepsilon_{1}\right) \geq 0
$$

comme $f$ est positive, on a $A \geq 0$.

Ensuite comme $\varphi$ est strictement convexe dans $\bar{\Omega} \backslash B\left(0, \delta_{1}\right)$, il est facile de voir que $A>0$ pour $x$ dans cet ensemble et $\xi \in \mathbb{R}^{n} \backslash\{0\}$.

B. Le résultat essentiel de ce paragraphe est la:

Proposition II.4. L est une opérateur formellement auto-adjoint.

Preuve. Elle consiste à montrer que tout opérateur $P$ de la forme

$$
P=\sum_{i, j=1}^{n} \frac{\partial F}{\partial u_{i j}}\left(u_{i j}\right) \partial_{i} \partial_{j}
$$

où $u \in C^{3}(\bar{\Omega})$ est formellement auto-adjoint.

Posons $\Psi(x)=\operatorname{det} u_{i j}(x)$ et $S=\{x \in \Omega, \Psi(x)=0\}$. On a

$$
P=\sum_{i=1}^{n} \partial_{i}\left(\sum_{j=1}^{n} \frac{\partial F}{\partial u_{i j}}\left(u_{i j}\right) \partial_{j}\right)-\sum_{i, j=1}^{n} \partial_{i}\left(\frac{\partial F}{\partial u_{i j}}\right)\left(u_{i j}\right) \partial_{j} ;
$$

nous allons montrer que pour tous $j=1, \ldots, n, x \in \bar{\Omega}$

$$
A_{j}(x)=\sum_{i=1}^{n} \partial_{i}\left(\frac{\partial F}{\partial u_{i j}}\right)\left(u_{i j}\right)(x)=\sum_{i, p, q=1}^{n} \frac{\partial^{2} F}{\partial u_{i j} \partial u_{p q}}\left(u_{i j}\right) u_{i p q}(x)=0 .
$$

Nous utiliserons l'identité suivante dont la preuve se trouve dans [ $\mathbf{Z}]$ (Lemme 1.6). Pour tous $i, j, p, q=1, \ldots, n$ on a

$$
\Psi \frac{\partial^{2} F}{\partial u_{i j} \partial u_{p q}}\left(u_{i j}\right)=\left(\frac{\partial F}{\partial u_{i j}} \frac{\partial F}{\partial u_{p q}}-\frac{\partial F}{\partial u_{i p}} \frac{\partial F}{\partial u_{j q}}\right)\left(u_{i j}\right) .
$$

Soit $x_{0} \in \bar{\Omega}$. Si $x_{0} \in S$ alors le résultat découle de (II.12), sinon on s'y ramène en utilisant essentiellement la continuité de la fonction déterminant.

C. L'objet de ce paragraphe est de rappeler certaines estimations classiques que nous utiliserons dans la suite, puis de prouver des estimations sur $G$ et l'opérateur $L_{G}(w)$. 
Soit $s_{*}$ un entier, $s_{*} \geq 7+n$. Il existe une constante $\beta>0$ (on prendra $\beta \geq 2$ ) telle que pour tous $0 \leq i, j, k \leq s_{*}+2$, pour $n_{*}=\frac{n}{2}+\kappa$ et $u \in C^{s_{*}+2, \alpha}(\Omega)$ on ait: Inégalité de Sobolev

$$
|u|_{i, \kappa} \leq \beta\|u\|_{i+n_{*}} .
$$

Inégalité de Gagliardo-Nirenberg

$$
\|u\|_{j} \leq \beta\|u\|_{i}^{\frac{k-j}{k-i}}\|u\|_{k}^{\frac{j-i}{k-i}}, \quad i<j<k .
$$

Opérateurs de lissage: pour tout $\lambda \geq 1$, il existe un opérateur $S_{\lambda}: H^{i}(\Omega) \rightarrow H^{j}(\Omega)$ tel que

$$
\begin{array}{ll}
\left\|S_{\lambda} u\right\|_{i} \leq \beta\|u\|_{j}, & i \leq j, \\
\left\|S_{\lambda} u\right\|_{i} \leq \beta \lambda^{i-j}\|u\|_{j}, & i \geq j, \\
\left\|S_{\lambda} u-u\right\|_{i} \leq \beta \lambda^{i-j}\|u\|_{j}, & i \leq j .
\end{array}
$$

On a aussi pour $u \in C^{s_{*}}(\Omega)$ :

$$
\|u\|_{s_{*}} \leq \beta|u|_{s_{*}} .
$$

On rappelle également les estimations suivantes (cf. A.G], Hör])

a) Si $u, v \in L^{\infty} \cap H^{s}(s>0)$ alors $u v \in L^{\infty} \cap H^{s}$ et il existe une constante $K_{1}>0$ indépendante de $u$ et $v$ telle que

$$
\|u v\|_{s} \leq K_{1}\left(|u|_{0}\|v\|_{s}+\|u\|_{s}|v|_{0}\right) .
$$

b) Soit $H: \mathbb{R}^{n} \rightarrow \mathbb{C}$, une fonction de classe $C^{\infty}$, vérifiant $H(0)=0$.

- Si $u \in\left(L^{\infty} \cap H^{s}\right)^{N}(s>0)$ alors $H(u) \in L^{\infty} \cap H^{s}$ et si $|u|_{0} \leq M$, il existe une constante $K_{2}=K_{2}(s, H, M)$ telle que:

$$
\|H(u)\|_{s} \leq K_{2}\|u\|_{s} .
$$

- Si $\left.u \in C^{i, \mu}, \mu \in\right] 0,1\left[, i \in \mathbb{N}\right.$ alors $H(u) \in C^{i, \mu}$ et si $|u|_{0} \leq M$, il existe une constante $K_{3}=K_{3}(\mu, i, H, M)$ telle que

$$
|H(u)|_{i, \mu} \leq K_{3}|u|_{i, \mu} .
$$

Le résultat principal de ce paragraphe est la:

Proposition II.5. Il existe une constante $K_{0}>0$, telle que pour toutes fonctions $w^{i} \in C^{s_{*}+2, \kappa}(\Omega),\left|w^{i}\right|_{2} \leq 1, i=1,2,3$ et pour $\varepsilon \leq 1$ on ait:

$$
\left|G\left(w^{1}\right)-G\left(w^{2}\right)\right|_{0} \leq K_{0}\left|w^{1}-w^{2}\right|_{2} *\left(\|\varphi\|_{2+n_{*}}+\left\|w^{2}\right\|_{2+n_{*}}+\left\|w^{1}\right\|_{2+n_{*}}\right)
$$

et pour tous $t \in[0,1], s \in\left[0, s_{*}\right]$ :

$$
\begin{aligned}
&\left\|\frac{d}{d t}\left[L_{G}\left(w^{1}+t w^{2}\right) w^{3}\right]\right\|_{s} \\
& \leq \varepsilon K_{0}\left[\left(\|\varphi\|_{s+2}+\varepsilon\left\|w^{1}\right\|_{s+2}+\varepsilon\left\|w^{2}\right\|_{s+2}+1\right)\left|w^{2}\right|_{2}\left|w^{3}\right|_{2}\right. \\
&+\left(\|\varphi\|_{2+n_{*}}+\varepsilon\left\|w^{1}\right\|_{2+n_{*}}+\varepsilon\left\|w^{2}\right\|_{2+n_{*}}+1\right) \\
&\left.\cdot\left(\left|w^{2}\right|_{2}\left\|w^{3}\right\|_{s+2}+\left|w^{3}\right|_{2}\left\|w^{2}\right\|_{s+2}\right)\right] .
\end{aligned}
$$


Preuve de (II.22).

(II.24)

$$
\begin{aligned}
G\left(w^{1}\right)-G\left(w^{2}\right) & =\frac{1}{\varepsilon}\left[\operatorname{det}\left(\varphi_{i j}+\varepsilon w_{i j}^{1}\right)-\operatorname{det}\left(\varphi_{i j}+\varepsilon w_{i j}^{2}\right)\right] \\
& =\int_{0}^{1} \sum_{i, j=1}^{n} \frac{\partial F}{\partial u_{i j}}\left(\varphi_{i j}+\varepsilon w_{i j}^{2}+t \varepsilon\left(w_{i j}^{1}-w_{i j}^{2}\right)\right)\left(w_{i j}^{1}-w_{i j}^{2}\right) d t .
\end{aligned}
$$

Or

$$
\left|\varphi_{i j}+\varepsilon w_{i j}^{2}+t \varepsilon\left(w_{i j}^{1}-w_{i j}^{2}\right)\right|_{0} \leq|\varphi|_{2}+2\left|w^{2}\right|_{2}+\left|w^{1}\right|_{2} \leq 3+|\varphi|_{2} .
$$

(II.22) résulte alors de (II.13), (II.21), (II.24) et (II.25).

Preuve de (II.23).

$$
\begin{aligned}
\frac{d}{d t}\left[L_{G}\left(w^{1}+t w^{2}\right) w^{3}\right] & =\frac{d}{d t}\left[\sum_{i, j=1}^{n} \frac{\partial F}{\partial u_{i j}}\left(\varphi_{i j}+\varepsilon w_{i j}^{1}+t \varepsilon w_{i j}^{2}\right) w_{i j}^{3}\right] \\
& =\varepsilon \sum_{i, j, p, q=1}^{n} \frac{\partial^{2} F}{\partial u_{i j} \partial u_{p q}}\left(\varphi_{i j}+\varepsilon w_{i j}^{1}+t \varepsilon w_{i j}^{2}\right) w_{p q}^{2} w_{i j}^{3} .
\end{aligned}
$$

D'après (ㅍ.19) on a pour $s \in\left[0, s_{*}\right]$ :

$$
\begin{aligned}
&\left\|\frac{d}{d t}\left[L_{G}\left(w^{1}+t w^{2}\right) w^{3}\right]\right\|_{s} \\
& \leq \varepsilon K_{1}\left[\sum_{i, j, p, q=1}^{n}\left\|\frac{\partial^{2} F}{\partial u_{i j} \partial u_{p q}}\left(\varphi_{i j}+\varepsilon w_{i j}^{1}+t \varepsilon w_{i j}^{2}\right)\right\|_{s}\left|w^{2}\right|_{2}\left|w^{3}\right|_{2}\right. \\
&\left.+K_{1}\left(\left|w^{2}\right|_{2}\left\|w^{3}\right\|_{s}+\left|w^{3}\right|_{2}\left\|w^{2}\right\|_{s}\right) \sum_{i, j, p, q=1}^{n}\left|\frac{\partial^{2} F}{\partial u_{i j} \partial u_{p q}}\left(\varphi_{i j}+\varepsilon w_{i j}^{1}+t \varepsilon w_{i j}^{2}\right)\right|_{0}\right] .
\end{aligned}
$$

Or

$$
\left|\left(\varphi_{i j}+\varepsilon w_{i j}^{1}+t \varepsilon w_{i j}^{2}\right)\right|_{0} \leq|\varphi|_{2}+\varepsilon\left|w^{1}\right|_{2}+\varepsilon t\left|w^{2}\right|_{2} \leq 2+|\varphi|_{2} .
$$

(II.23) résulte alors de (II.13), (II.20) et (II.21).

II.2. Existence de solutions et estimations $H^{s}$. Soit $\varepsilon>0$ satisfaisant aux contraintes apparaissant aux Lemme $\Pi .2$ Propositions $\llbracket .3$ et $\llbracket .5$; et $L$ l'opérateur défini en (II.10).

$$
\begin{aligned}
L & =-L_{G}(w)-\theta \Delta=\sum_{i, j=1}^{n} a^{i j} \partial_{i} \partial_{j}, \\
a^{i j} & =-\frac{\partial F}{\partial u_{i j}}\left(\varphi_{i j}+\varepsilon w_{i j}\right)-\theta \delta_{i j}=-\Phi^{i j}-\theta \delta_{i j} .
\end{aligned}
$$

Pour $k, s \in \mathbb{N}$ on note:

$$
\left\{\begin{array}{l}
A(k)=\max \left(1, \max _{1 \leq i, j \leq n}\left|a^{i j}\right|_{k}\right) \text { et } \\
\Lambda_{s}=\{(i, j), 0 \leq i, j \leq s, i+j \leq s \text { et } i+2 \leq \max (s, 2)\} .
\end{array}\right.
$$

Le résultat principal de ce paragraphe est le suivant: 
Théorème II.6. Supposons que $\theta \leq 1$ et qu'il existe $M_{0}>0$ tel que $A(2) \leq M_{0}$. Il existe alors $\varepsilon_{3}>0$ tel que pour tout $\varepsilon, 0<\varepsilon \leq \varepsilon_{3}$, tout $w \in C^{s_{*}+2, \kappa}(\Omega)$ vérifiant $|w|_{3, \kappa} \leq 1$ et tout $g \in H^{s_{*}}$, le problème

$$
\left\{\begin{array}{l}
L u=g \text { dans } \Omega \\
\left.u\right|_{\partial \Omega}=0
\end{array}\right.
$$

possède une solution unique $u \in H^{s_{*}}$. De plus pour tout $0 \leq s \leq s_{*}$ il existe une constante $C_{s}>0, C_{s}=C_{s}\left(\varphi, s, \Omega, M_{0}, \varepsilon_{3}\right)$ telle que

$$
\begin{aligned}
& \|u\|_{0} \leq C_{0}\|g\|_{0}, \\
& \|u\|_{1} \leq C_{1}\left(\|g\|_{1}+\|u\|_{0}\right), \\
& \|u\|_{s} \leq C_{s}\left(\|g\|_{s}+\sum_{\substack{(i, j) \in \Lambda_{s} \\
j \leq s-1}}\left(1+|\varphi+\varepsilon w|_{i+4, \kappa}\right)\|u\|_{j}\right), \quad s \geq 2 .
\end{aligned}
$$

Pour montrer ce théorème on va d'abord montrer la proposition suivante. Posons pour $\nu \in] 0,1], L_{\nu}=L-\nu \Delta$.

Proposition II.7. Supposons que $\theta \leq 1$ et qu'il existe $M_{0}>0$ tel que $A(2) \leq M_{0}$. Il existe alors $\varepsilon_{3}>0$ tel que pour tous $0<\varepsilon \leq \varepsilon_{3}$,w $\in C^{s_{*}+2, \kappa}(\Omega)$ vérifiant $|w|_{3, \kappa} \leq 1$ et $g \in H^{s_{*}}(\Omega)$, le problème

$$
\left\{\begin{array}{l}
L_{\nu} u=g \text { dans } \Omega \\
\left.u\right|_{\partial \Omega}=0
\end{array}\right.
$$

possède une solution unique $u \in H^{s_{*}+1}(\Omega)$.

Preuve. D'après la Proposition 11.3 , $L$ a un symbole positif ou nul donc $L_{\nu}$ est uniformément elliptique. Les coefficients de $L_{\nu}$ sont dans $C^{s_{*}-1,1}(\Omega)$ et $g \in H^{s_{*}}$ donc d'après un résultat de Gilbarg, Trudinger (G.T], Théorèmes 6.14 et 8.13), le problème $(\mathrm{II} .28)^{\prime}$ possède une solution unique $u \in H^{s_{*}+1}(\Omega)$.

Le reste de ce paragraphe est consacré à montrer les estimations (II.29) à (II.31) pour l'opérateur régularisé $L_{\nu}$ avec des constantes $C_{s}$ indépendantes de $\nu$. Puis en faisant tendre $\nu$ vers zéro on obtient la solution $u \in H^{s_{*}}(\Omega)$ de (II.28) et les estimations (II.29) à (II.31). Pour la preuve on va adopter la technique de [A] au cas présent.

II.2.1. Estimations dans la zone elliptique de L. Les résultats de ce paragraphe que nous reproduisons pour la commodité du lecteur, sont prouvés par Amano [A].

Proposition II.8 ([₫] , Lemme 3.9). Soit $P=\sum_{i, j=1}^{n} a^{i j} \partial_{i} \partial_{j}$ un opérateur à coefficients réels appartenant à $C^{s_{*}, \kappa}(\Omega)$. On suppose que $P$ est uniformément elliptique dans $\bar{\Omega}$, c'est-à-dire qu'il existe une constante $\lambda_{0}>0$ telle que:

$$
\sum_{i, j=1}^{n} a^{i j} \xi_{i} \xi_{j} \geq \lambda_{0}|\xi|^{2}, \text { pour tout }(x, \xi) \in \bar{\Omega} \times \mathbb{R}^{n} .
$$

Alors pour tout entier $1 \leq s \leq s_{*}$ il existe une constante $C_{s}^{\prime}$ qui ne dépend que de $s, \lambda_{0}, \Omega$ et de la norme $L^{\infty}$ des coefficients de $P$, telle que pour toute fonction réelle 
$u \in C^{s_{*}, \kappa}(\Omega) \cap H_{0}^{1}(\Omega)$ on ait:

$$
\begin{aligned}
& \|u\|_{1} \leq C_{1}^{\prime}\left(\|P u\|_{0}+A(2)\|u\|_{0}\right), \\
& \|u\|_{s} \leq C_{s}^{\prime}\left(\|P u\|_{s-1}+\sum_{\substack{i+j \leq s-1 \\
i \leq s-2}} A(i+2)\|u\|_{j}\right), \quad s \geq 2 .
\end{aligned}
$$

D'autre part soit $P=\sum_{i, j=1}^{n} a^{i j} \partial_{i} \partial_{j}$ un opérateur à coefficients réels appartenant à $C^{s_{*}, \kappa}(\Omega)$, dont le symbole est positif ou nul. Soit $\lambda(x)$ la plus petite valeur propre de la matrice $\left(a^{i j}(x)\right)$. Notons $S=\{x \in \Omega, \lambda(x)=0\}$ et pour $\delta>0, S_{\delta}=\{x \in \Omega, d(x, \Omega)<\delta\}$. On a:

Lemme II.9 ( $\underline{\mathrm{A}}$, Lemme 3.2). Supposons $P$ auto-adjoint et $S$ réduit à un point. Il exite une fonction $\mu \in L^{\infty}(\Omega)$ et une constante $C>0$, telle que $\mu=0$ sur $S$, $\inf _{\bar{\Omega} \backslash S_{\delta}} \mu>0$ pour $\delta$ assez petit et telle que pour $u \in C^{s_{*}, \kappa}(\Omega) \cap H_{0}^{1}(\Omega)$ on ait

$$
\int_{\Omega} \mu u^{2} d x+\int_{\Omega} \lambda|\nabla u|^{2} d x \leq C\|P u\|_{0}\|u\|_{0} .
$$

II.2.2. Estimation au voisinage du lieu de dégénérescence de $L$. Soit $L_{\nu}=L-\nu \Delta$ où $L$ est défini en (II.26). Rappelons que d'après la Proposition 【I.4, il est autoadjoint.

Pour $t \geq 1$ posons $U(t)=\left\{x \in \Omega,\left|x_{n}\right|<\frac{1}{t}\right\}$ et $V(t)=U(t) \cap B\left(0, \delta_{1}\right)$ où $\delta_{1}$ est défini dans le Lemme $\llbracket .2$

Le résultat principal de ce paragraphe est la:

Proposition II.10. Pour tout entier $0 \leq s \leq s_{*}$ et toute fonction $u \in C_{0}^{s_{*}, \kappa}(V(t))$, il existe $C_{s}^{\prime \prime}=C_{s}^{\prime \prime}\left(n, \Omega, \varphi, \delta_{1}\right)>0$ telle que

$$
\begin{aligned}
& \|u\|_{0} \leq C_{0}^{\prime \prime} t^{-2}\left\|L_{\nu} u\right\|_{0}, \\
& \|u\|_{s} \leq C_{s}^{\prime \prime} t^{-2}\left(\left\|L_{\nu} u\right\|_{s}+\sum_{(i, j) \in \Lambda_{s}} A(i+2)\|u\|_{j}\right), \quad s \geq 1 .
\end{aligned}
$$

Preuve de (II.35). Elle utilise l'inégalité (II.7) et la généralisation en dimension $n$ d'un lemme d'Amano (cf. [A], Lemme 3.1).

Preuve de (II.36). Elle se fait par récurrence en utilisant (II.35).

\section{II.2.3. Estimation des commutateurs.}

Lemme II.11 ([ब] , Lemme 3.7). Soit $P=\sum_{i, j=1}^{n} a^{i j} \partial_{i} \partial_{j}$ un opérateur à coefficients réels appartenant à $C^{s_{*}}(\Omega)$ dont le symbole est positif ou nul. Soit $\chi \in$ $C^{\infty}(\bar{\Omega})$ telle que Supp $\nabla \chi \subset \Omega$. Alors pour tout entier $0 \leq s \leq s_{*}$, il existe une constante $C_{s}>0$ telle que pour $u \in C^{s_{*}, \kappa}(\Omega)$

$$
\|[\chi, P] u\|_{s} \leq C_{s}\left(\|P u\|_{s}+\sum_{(i, j) \in \Lambda_{s}} A(i+2)\|u\|_{j}\right) .
$$


II.2.4. Preuve des estimations (II.29) à (II.31) pour $L_{\nu}$. Soient $\chi \in C_{0}^{\infty}(V(t))$, $\chi_{1}, \chi_{2} \in C_{0}^{\infty}(\bar{\Omega} \backslash\{0\}), 0 \leq \chi, \chi_{1}, \chi_{2} \leq 1$ et telles que $\chi=1$ dans un voisinage de zéro contenu dans $V(t), \chi_{1}=1$ sur le support de $\partial_{i} \chi$ pour tout $i$ et $\chi_{2}=1$ sur le support de $\chi_{1}$.

Soit $u \in C^{s_{*}, \kappa}(\Omega) \cap H_{0}^{1}$. Comme Supp $\chi \subset V(t)$, on a d'après (II.36) pour $1 \leq s \leq s_{*}$ :

$$
\begin{aligned}
\|\chi u\|_{s} \leq & C_{s}^{\prime \prime} t^{-2}\left(\left\|\chi L_{\nu} u\right\|_{s}+\left\|\left[\chi, L_{\nu}\right]\right\|_{s}+\sum_{\substack{(i, j) \in \Lambda_{s} \\
j<s}}(A(i+2)+1)\|u\|_{j}\right) \\
& +C_{s}^{\prime \prime} t^{-2}(A(2)+1)\|\chi u\|_{s} .
\end{aligned}
$$

Par hypothèse $A(2) \leq M_{0}$. On fixe donc $t$ tel que pour $1 \leq s \leq s_{*}$

$$
C_{s}^{\prime \prime} t^{-2}\left(M_{0}+1\right) \leq \frac{1}{2}
$$

D'autre part $A(i+2)=\max \left(1, \max _{1 \leq p, q \leq n}\left|\frac{\partial F}{\partial u_{p q}}\left(\varphi_{k l}+\varepsilon w_{k l}\right)\right|_{i+2}+\theta\right)$ or $\left|\varphi_{k l}+\varepsilon w_{k l}\right|_{0} \leq|\varphi|_{2}+1$, il résulte donc de (II.21) que pour $0 \leq i \leq s_{*}-2,1 \leq p, q \leq n$ :

$$
\left|\frac{\partial F}{\partial u_{p q}}\left(\varphi_{k l}+\varepsilon w_{k l}\right)\right|_{i+2} \leq C(\varphi)|\varphi+\varepsilon w|_{i+4, \kappa},
$$

d'où comme $\theta \leq 1$ :

$$
A(i+2) \leq 1+C(\varphi)|\varphi+\varepsilon w|_{i+4, \kappa} .
$$

On déduit de (II.38), (II.39) et (II.40)

$$
\|\chi u\|_{s} \leq 2 C_{s}^{\prime \prime} t^{-2}\left(\left\|L_{\nu} u\right\|_{s}+\left\|\left[\chi, L_{\nu}\right]\right\|_{s}+\sum_{\substack{(i, j) \in \Lambda_{s} \\ j<s}}\left(1+|\varphi+\varepsilon w|_{i+4, \kappa}\right)\|u\|_{j}\right) .
$$

Preuve de (II.29). Comme $\chi=1$ dans un voisinage de zéro dans $V(t)$, il existe $\delta>0$ tel que $\operatorname{Supp}(1-\chi) \subset \bar{\Omega} \backslash B(0, \delta)$. Considérons la fonction $\mu$ définie dans le Lemme $\llbracket$ et notons $m=\inf _{\bar{\Omega} \backslash B(0, \delta)} \mu>0$. Remarquons que $m$ ne dépend que de $\varphi, \Omega, n$.

D'après (II.34), il existe $C_{0}=C_{0}(\varphi, \Omega, n)>0$ tel que:

$$
\|(1-\chi) u\|_{0}^{2}=\int_{\bar{\Omega} \backslash B(0, \delta)} u^{2} d x \leq \frac{1}{m} \int \mu u^{2} d x \leq C_{0}\left\|L_{\nu} u\right\|_{0}\|u\|_{0} .
$$

Puisque Supp $\chi_{2} \subset \bar{\Omega} \backslash\{0\}$ on a de même

$$
\left\|\chi_{2} u\right\|_{0}^{2} \leq C_{1}\left\|L_{\nu} u\right\|_{0}\|u\|_{0} .
$$

D'autre part d'après (II.35)

$$
\|\chi u\|_{0}^{2} \leq C_{2}\left\|L_{\nu} \chi u\right\|_{0}^{2} \leq C_{2}\left(\left\|L_{\nu} u\right\|_{0}^{2}+\left\|\left[\chi, L_{\nu}\right] u\right\|_{0}^{2}\right) .
$$

Or on a $\chi_{1} L_{\nu} \chi_{2} u=\chi_{1} L_{\nu} u$ et $\left[\chi, L_{\nu}\right] u=\left[\chi, \chi_{1} L_{\nu}\right] \chi_{2} u$, d'où d'après le Lemme et comme $A(2) \leq M_{0}$ et $\nu \leq 1$ :

$$
\begin{aligned}
\left\|\left[\chi, L_{\nu}\right] u\right\|_{0}^{2}=\left\|\left[\chi, \chi_{1} L_{\nu}\right] \chi_{2} u\right\|_{0}^{2} & \leq C\left(\left\|\chi_{1} L_{\nu} \chi_{2} u\right\|_{0}^{2}+\left(M_{0}+1\right)^{2}\left\|\chi_{2} u\right\|_{0}^{2}\right) \\
& \leq C^{\prime}\left(\left\|L_{\nu} u\right\|_{0}^{2}+\left\|\chi_{2} u\right\|_{0}^{2}\right) .
\end{aligned}
$$


En combinant (II.42) à (II.45) on obtient (II.29).

Preuve de (II.30). On a $\operatorname{supp}(1-\chi) \subset \bar{\Omega} \backslash B(0, \delta)$. Or $\varphi$ est strictement convexe sur le support de $(1-\chi)$, donc $L$ est uniformément elliptique sur cet ensemble. D'après (II.32) et le fait que $A(2) \leq M_{0}$ on a

$$
\|(1-\chi) u\|_{1} \leq C_{1}^{\prime}\left(\left\|L_{\nu} u\right\|_{0}+\left(M_{0}+1\right)\|u\|_{0}+\left\|\left[\chi, L_{\nu}\right] u\right\|_{0}\right) .
$$

En appliquant le Lemme II.11 on obtient:

$$
\left\|\left[\chi, L_{\nu}\right] u\right\|_{0} \leq C_{0}\left(\left\|L_{\nu} u\right\|_{0}+\left(M_{0}+1\right)\|u\|_{0}\right) .
$$

On en déduit

$$
\|(1-\chi) u\|_{1} \leq C_{1}\left(M_{0}\right)\left(\left\|L_{\nu} u\right\|_{0}+\|u\|_{0}\right) .
$$

D'autre part (II.41) et $A(2) \leq M_{0}$ impliquent:

$$
\|\chi u\|_{1} \leq C\left(M_{0}\right)\left(\left\|L_{\nu} u\right\|_{1}+\left\|\left[\chi, L_{\nu}\right]\right\|_{1}+\|u\|_{0}\right) .
$$

Comme $\left[\chi, L_{\nu}\right] u=\left[\chi, \chi_{1} L_{\nu}\right] \chi_{2} u$ et $\chi_{1} L_{\nu} \chi_{2} u=\chi_{1} L_{\nu} u$, le Lemme II.11 et $A(2) \leq$ $M_{0}$ donnent:

$$
\begin{aligned}
\left\|\left[\chi, L_{\nu}\right] u\right\|_{1} & \leq C_{1}\left(\left\|\chi_{1} L_{\nu} \chi_{2} u\right\|_{1}+\left(1+M_{0}\right)\left\|\chi_{2} u\right\|_{1}\right) \\
& \leq C_{1}\left(\left\|L_{\nu} u\right\|_{1}+\left(M_{0}+1\right)\left\|\chi_{2} u\right\|_{1}\right) .
\end{aligned}
$$

Or $L$ est uniformément elliptique sur le support de $\chi_{2}$, on a donc d'après (II.32) et $A(2) \leq M_{0}$ :

$$
\left\|\chi_{2} u\right\|_{1} \leq C_{1}^{\prime}\left(\left\|L_{\nu} u\right\|_{0}+\left(M_{0}+1\right)\|u\|_{0}+\left\|\left[\chi_{2}, L_{\nu}\right] u\right\|_{0}\right)
$$

et en utilisant (II.38) on a:

$$
\left\|\left[\chi_{2}, L_{\nu}\right] u\right\|_{0} \leq C\left(M_{0}\right)\left(\left\|L_{\nu} u\right\|_{0}+\|u\|_{0}\right)
$$

d'où

$$
\|\chi u\|_{1} \leq C_{1}\left(M_{0}\right)\left(\left\|L_{\nu} u\right\|_{1}+\|u\|_{0}\right) .
$$

(II.46) et (II.47) donnent (II.30).

Preuve de (II.31). Elle est identique à celle de (II.30) et s'obtient en utilisant les inégalités (II.33), (II.40) et (II.41).

II.3. Schéma itératif du type Nash-Moser. Dans ce paragraphe on va utiliser un schéma itératif du type Nash-Moser et les résultats du paragraphe II.2 pour prouver le Théorème $\mathrm{A}$

Il est auparavant nécessaire de fixer les constantes $\varepsilon_{0}$ et $M_{0}$ apparaissant dans Théorèmes A et (II.6).

On pose

$$
M_{0}=1+\max _{1 \leq i, j \leq n} K_{3}\left(2, \kappa, \frac{\partial F}{\partial u_{i j}},\left(1+|\varphi|_{2}\right)\right)\left(1+|\varphi|_{4, \kappa}\right)
$$

où $K_{3}$ est la constante intervenant dans l'inégalité (II.21). $M_{0}$ étant fixé on pose

$$
D=\max \left(\max _{0 \leq s \leq s_{*}} C_{s}, 1\right)
$$

où $C_{s}$ est la constante (ne dépendant que de $s, \varphi, \Omega, M_{0}$ ) déterminée dans le Théorème II.6. Ensuite

$$
\mu=\max \left(\beta, 3 D s_{*}^{2}\left(1+|\varphi|_{s_{*}+2, \kappa}\right), 2^{1 / \kappa}\right) \text { et } \bar{\mu}=\beta^{2} \mu^{s_{*}},
$$


puis

$$
\left\{\begin{array}{l}
a_{1}=9 K_{0} \mu^{5}, \\
a_{2}=5 a_{1} \mu^{s_{*}+1}, \\
a_{3}=7 K_{0} \mu^{5},
\end{array}\right.
$$

où $K_{0}$ est la constante déterminée dans la Proposition 1 I.5.

Enfin on fixe $\bar{\varepsilon}$ vérifiant

$$
\bar{\varepsilon} \leq \inf _{1 \leq i \leq 4}\left(1, \varepsilon_{i}\right)
$$

où les $\varepsilon_{i}$ sont déterminés dans les Lemme $\llbracket 1.2$ Proposition $\llbracket .3$ Théorème $\llbracket .6$ et la preuve de (II.30), de plus

$$
\bar{\varepsilon} \leq\left(3 D^{2} a_{2}+6 \bar{\mu} D^{2}\right)^{-2} .
$$

Ces contraintes étant déterminées, soit $f \in C^{s_{*}}(\Omega)$ telle que

$$
\left|\operatorname{det} \varphi_{i j}-f\right|_{s_{*}} \leq \bar{\varepsilon}^{2} .
$$

Le $\varepsilon_{0}$ mentionné dans le Théorème $\mathrm{A}$ est alors égal à $\bar{\varepsilon}^{2}$. Le schéma formel de la méthode est le suivant. On pose tout d'abord pour $n \in \mathbb{N}, \mu_{n}=\mu^{n}$ où $\mu$ est défini dans (II.50) puis $S_{n}=S \mu_{n}$, l'opérateur de lissage défini dans le paragraphe C. Ensuite pour $n \in \mathbb{N}$

$$
u_{0}=0, w_{0}=0, w_{n+1}=w_{n}+u_{n+1}, \quad n \geq 0,
$$

$u_{n+1}$ étant la solution du problème de Dirichlet

$$
L_{G}\left(\tilde{w}_{n}\right) u_{n+1}+\theta_{n} \Delta u_{n+1}=f_{n} \text { dans } \Omega,\left.\quad u_{n+1}\right|_{\partial \Omega}=0,
$$

où

$$
\begin{gathered}
\tilde{w}_{n}=S_{n} w_{n}, \\
\theta_{n}=\left|G\left(\tilde{w}_{n}\right)\right|_{0}, \\
f_{0}=-S_{0} G(0), f_{n}=S_{n-1} R_{n-1}-S_{n} R_{n}+S_{n-1} G(0)-S_{n} G(0), \\
R_{0}=0, \quad R_{n}=\sum_{j=1}^{n} r_{j}, \\
r_{0}=0, r_{j}=\left[L_{G}\left(w_{j-1}\right)-L_{G}\left(\tilde{w}_{j-1}\right)\right] u_{j}+Q_{j}-\theta_{j-1} \Delta u_{j}, \quad 1 \leq j \leq n, \\
Q_{j}=G\left(w_{j}\right)-G\left(w_{j-1}\right)-L_{G}\left(w_{j-1}\right) u_{j}, \quad 1 \leq j \leq n .
\end{gathered}
$$

Ce schéma formel est cohérent puisque la connaissance de $u_{j}$ pour $1 \leq j \leq n$ détermine celle de $w_{n}$ donc de $\tilde{w}_{n}$ et $\theta_{n}$ puis celle de $r_{j}$ pour $1 \leq j \leq n$ donc celle de $R_{n}$ et de $f_{n},(\text { II. } 56)_{n+1}$ permet alors de déterminer $u_{n+1}$. L'existence de la suite $u_{n}$ et la convergence de $w_{n}$ vers une solution $w$ de $G(w)=0,\left.w\right|_{\partial \Omega}=0$, découleront de la proposition suivante.

Proposition II.12. Soit $s_{*}$ un entier, $s_{*} \geq 7+n$. Fixons $\sigma$ tel que $4+n+2 \kappa \leq$ $\sigma<s_{*}-2$. Pour tout $n \in \mathbb{N}$, le problème (II.56) $)_{n+1}$ admet une solution unique 
$u_{n+1} \in H^{s_{*}}(\Omega)$. De plus pour tout $s \in \mathbb{N}$ on $a$ :

$$
\begin{gathered}
\left\|u_{j}\right\|_{s} \leq \sqrt{\bar{\varepsilon}}\left[\max \left(\mu, \mu_{j-1}\right)\right]^{s-\sigma}, \quad j \in \mathbb{N}^{*}, \quad 0 \leq s \leq s_{*}, \\
\left\|w_{j}\right\|_{s} \leq\left\{\begin{array}{l}
2 \sqrt{\bar{\varepsilon}}, \quad s \leq \sigma-\kappa, \\
\sqrt{\bar{\varepsilon}} \mu_{j}^{s-\sigma}, \quad \sigma+\kappa \leq s \leq s_{*}, \quad j \in \mathbb{N}^{*}, \\
\left|\tilde{w}_{j}\right|_{4, \kappa} \leq 1, \quad j \in \mathbb{N}^{*},
\end{array}\right. \\
\left\|w_{j}-\tilde{w}_{j}\right\|_{s} \leq 2 \beta \sqrt{\bar{\varepsilon}} \mu_{j}^{s-\sigma}, \quad 0 \leq s \leq s_{*}, \quad j \in \mathbb{N}^{*}, \\
\left\|r_{j}\right\|_{s} \leq \bar{\varepsilon} a_{1}\left[\max \left(\mu, \mu_{j-1}\right)\right]^{s-\sigma}, \quad 0 \leq s \leq s_{*}-2, \quad j \in \mathbb{N}^{*}, \\
f_{j} \in H^{s_{*}} \text { et }\left\|f_{j}\right\|_{s} \leq \bar{\varepsilon} a_{2} \mu_{j}^{s-\sigma}, \quad 0 \leq s \leq s_{*}, \quad j \in \mathbb{N}, \\
\theta_{j} \leq a_{3} \sqrt{\bar{\varepsilon}} \mu_{j}^{-2} \leq 1, \quad j \in \mathbb{N}, \\
A_{j}(2) \leq M_{0}, \quad j \in \mathbb{N} .
\end{gathered}
$$

Preuve. On a $u_{0}=0$, montrons que les inégalités (II.68) à (II.70) $)_{0}$ sont vérifiées.

a) (II.68) $)_{0}$ : D'après (III.8) et (II.59) on a:

$$
f_{0}=-S_{0} G(0) \text { et } G(0)=\frac{1}{\bar{\varepsilon}}\left(\operatorname{det} \varphi_{i j}-f\right) .
$$

Or $\varphi \in C^{s_{*}+2, \alpha}(\Omega), f \in C^{s_{*}}(\Omega)$ et les $S_{n}$ sont infiniment régularisants donc $f_{0} \in$ $H^{s_{*}}(\Omega)$. D'autre part d'après (II.8), (II.15), (II.18) et (II.54) on a:

$$
\left\|f_{0}\right\|_{s} \leq \beta\|G(0)\|_{s} \leq \frac{\beta}{\bar{\varepsilon}}\left\|\operatorname{det} \varphi_{i j}-f\right\|_{s_{*}} \leq \frac{\beta^{2}}{\bar{\varepsilon}}\left|\operatorname{det} \varphi_{i j}-f\right|_{s_{*}} \leq \beta^{2} \bar{\varepsilon} .
$$

(II.51) et $\beta \leq \mu$ impliquent:

$$
\left\|f_{0}\right\|_{s} \leq \mu^{2} \bar{\varepsilon} \leq a_{2} \bar{\varepsilon}
$$

b) $(\text { II.69) })_{0}$ : (II.8), (II.51), (II.53) et (II.54) donnent:

$$
\theta_{0}=|G(0)|_{0} \leq \frac{1}{\bar{\varepsilon}}\left|\operatorname{det} \varphi_{i j}-f\right|_{s_{*}} \leq \bar{\varepsilon} \leq \sqrt{\bar{\varepsilon}} a_{3} \leq 1 .
$$

c) $(\text { II.70) })_{0}:$ On a $A_{0}(2)=\max \left(1, \max _{1 \leq i, j \leq n}\left|\frac{\partial F}{\partial \varphi_{i j}}\left(\varphi_{p q}\right)\right|_{2}+\theta_{0}\right)$. (II.13), (II.48) et (II.69) impliquent $A_{0}(2) \leq M_{0}$.

Supposons que l'on ait construit $u_{0}, u_{1}, \ldots, u_{n-1} \in H^{s_{*}}(\Omega)$ vérifiant (II.29) à (II.31) et que l'on ait montré les inégalités (II.63) $)_{j}$ à (II.70) $)_{j}$ pour $0 \leq j \leq n-$ 1. On va alors construire $u_{n} \in H^{s_{*}}(\Omega)$ vérifiant (II.29) à (II.31) puis montrer que (II.63) $)_{n}$ à $(\text { II.70) })_{n}$ sont vérifiées. Il résulte de (II.72) $)_{n-1}$ à (II.79) $)_{n-1}$ que $\left|\tilde{w}_{n-1}\right|_{4, \kappa} \leq 1, \theta_{n-1} \leq 1, A_{n-1}(2) \leq M_{0}$ et $f_{n-1} \in H^{s_{*}}(\Omega)$. On peut donc appliquer le Théorème \I.6 et obtenir la solution $u_{n} \in H^{s_{*}}(\Omega)$ de (II.56) $)_{n}$ vérifiant (II.29) à (II.31). Ensuite:

a) $(\text { II.63) })_{n}$ : • Cas $n=1$ : D'après (II.15), (III.18), (III.29), (II.49) et (II.54) on a

$$
\left\|u_{1}\right\|_{0} \leq D\left\|f_{0}\right\|_{0} \leq D \beta\|G(0)\|_{0} \leq D \frac{\beta^{2}}{\bar{\varepsilon}}\left|\operatorname{det} \varphi_{i j}-f\right|_{s_{*}} \leq D \beta^{2} \bar{\varepsilon} .
$$

(I.50), (ㅍ.53) et $s_{*} \geq \sigma$ impliquent

$$
\left\|u_{1}\right\|_{0} \leq D \overline{\mu \varepsilon} \mu^{-s_{*}}
$$

puis

$$
\left\|u_{1}\right\|_{0} \leq \sqrt{\bar{\varepsilon}} \mu^{-\sigma}
$$


Il résulte de (II.30) que:

$$
\left\|u_{1}\right\|_{1} \leq D\left(\left\|f_{0}\right\|_{1}+\left\|u_{1}\right\|_{0}\right) .
$$

On a alors d'après (III.71), (II.68) $)_{0}$ et $s_{*} \geq \sigma$ :

$$
\left\|u_{1}\right\|_{1} \leq D\left(\beta^{2} \bar{\varepsilon}+D \overline{\mu \varepsilon} \mu^{-\sigma}\right) \leq 2 D^{2} \overline{\mu \varepsilon} \mu^{-\sigma} .
$$

Donc en utilisant (II.53) on obtient

$$
\left\|u_{1}\right\|_{1} \leq \sqrt{\bar{\varepsilon}} \mu^{1-\sigma} .
$$

Supposons que l'on ait pour $0 \leq l<s, s \geq 2$ :

$$
\left\|u_{1}\right\|_{l} \leq \sqrt{\bar{\varepsilon}} \mu^{l-\sigma}
$$

montrons que $\left\|u_{1}\right\|_{s} \leq \sqrt{\bar{\varepsilon}} \mu^{s-\sigma}$.

D'après (II.31) on a pour $s \geq 2$ :

$$
\left\|u_{1}\right\|_{s} \leq D\left(\left\|f_{0}\right\|_{s}+\sum_{\substack{(i, l) \in \Lambda_{s} \\ l \leq s-1}}\left(1+|\varphi|_{i+4, \kappa}\right)\left\|u_{1}\right\|_{l}\right)
$$

(II.15), (II.18), (II.50), (II.54), (II.59) et $s_{*}+s \geq \sigma$ impliquent

$$
\left\|f_{0}\right\|_{s} \leq \beta\|G(0)\|_{s} \leq \beta^{2}|G(0)|_{s_{*}} \leq \beta^{2} \bar{\varepsilon}=\bar{\mu} \mu^{-s_{*}} \bar{\varepsilon} \leq \overline{\mu \varepsilon} \mu^{s-\sigma} .
$$

En utilisant (II.72), (II.73) on obtient:

$$
\begin{aligned}
\left\|u_{1}\right\|_{s} & \leq D\left(\overline{\mu \varepsilon} \mu^{s-\sigma}+\sum_{\substack{(i, l) \in \Lambda_{s} \\
l \leq s-1}}\left(1+|\varphi|_{s_{*}+2, \kappa}\right) \sqrt{\bar{\varepsilon}} \mu^{l-\sigma}\right) \\
& \leq D\left(\overline{\mu \varepsilon} \mu^{s-\sigma}+s_{*}^{2}\left(1+|\varphi|_{s_{*}+2, \kappa}\right) \mu^{-1} \sqrt{\bar{\varepsilon}} \mu^{s-\sigma}\right)
\end{aligned}
$$

(II.50) et (II.53) impliquent alors

$$
\left\|u_{1}\right\|_{s} \leq \sqrt{\bar{\varepsilon}} \mu^{s-\sigma} .
$$

- Cas $n \geq 2$ : (II.29) et (II.49), ([I.53) et (II.68) $)_{n-1}$ impliquent

$$
\left\|u_{n}\right\|_{0} \leq D\left\|f_{n-1}\right\|_{0} \leq D \bar{\varepsilon} a_{2} \mu_{n-1}^{-\sigma} \leq \sqrt{\bar{\varepsilon}} \mu_{n-1}^{-\sigma}
$$

De même (II.30), (II.49), (II.53), (II.68) $)_{n-1}$ et (II.75) donnent

$$
\left\|u_{n}\right\|_{1} \leq \sqrt{\bar{\varepsilon}} \mu_{n-1}^{1-\sigma} \text {. }
$$

Supposons que l'on ait pour $0 \leq l<s, s \geq 2$

$$
\left\|u_{n}\right\|_{l} \leq \sqrt{\bar{\varepsilon}} \mu_{n-1}^{l-\sigma} \text {. }
$$

D'après (II.31) on a

$$
\left\|u_{n}\right\|_{s} \leq D\left(\left\|f_{n-1}\right\|_{s}+\sum_{\substack{(i, l) \in \Lambda_{s} \\ l \leq s-1}}\left(1+\left|\varphi+\bar{\varepsilon} \tilde{w}_{n-1}\right|_{i+4, \kappa}\right)\|u\|_{l}\right) .
$$

(II.13), (II.16), (II.64) $)_{n-1}$ et $5+\left[n_{*}\right] \leq \sigma-\kappa$ impliquent pour $0 \leq i \leq s-2$

$$
\left|\tilde{w}_{n-1}\right|_{i+4, \kappa} \leq \beta\left\|\tilde{w}_{n-1}\right\|_{4+n_{*}+i} \leq \beta \mu_{n-1}^{i}\left\|w_{n-1}\right\|_{4+n_{*}} \leq 2 \beta^{2} \sqrt{\bar{\varepsilon}} \mu_{n-1}^{i} .
$$


On obtient alors en utilisant (II.76), (II.77) et (II.78)

$$
\begin{aligned}
\left\|u_{n}\right\|_{s} & \leq D\left(\bar{\varepsilon} a_{2} \mu_{n-1}^{s-\sigma}+\sum_{\substack{(i, l) \in \Lambda_{s} \\
l \leq s-1}}\left(1+|\varphi|_{s_{*}+2, \kappa}+2 \beta^{2} \sqrt{\bar{\varepsilon}} \mu_{n-1}^{i}\right) \sqrt{\bar{\varepsilon}} \mu_{n-1}^{l-\sigma}\right) \\
& \leq D\left(\bar{\varepsilon} a_{2} \mu_{n-1}^{s-\sigma}+2 s_{*}^{2} \beta^{2} \bar{\varepsilon} \mu_{n-1}^{s-\sigma}+\left(|\varphi|_{s_{*}+2, \kappa}+1\right) s_{*}^{2} \sqrt{\bar{\varepsilon}} \mu_{n-1}^{s-1-\sigma}\right)
\end{aligned}
$$

(II.50) et (II.53) impliquent alors:

$$
\left\|u_{n}\right\|_{s} \leq \sqrt{\bar{\varepsilon}} \mu_{n-1}^{s-\sigma} .
$$

b) (II.64) $)_{n}$ : (II.55) implique que $w_{n}=\sum_{j=1}^{n} u_{j}$. D'après (II.63) $j \leq j \leq n$ on

$$
\left\|w_{n}\right\|_{s} \leq \sum_{j=1}^{n}\left\|u_{j}\right\|_{s} \leq \sqrt{\bar{\varepsilon}} \mu^{s-\sigma}+\sum_{j=2}^{n} \sqrt{\bar{\varepsilon}} \mu_{j-1}^{s-\sigma} \leq \sqrt{\bar{\varepsilon}} \mu^{s-\sigma}+\sum_{j=1}^{n-1} \sqrt{\bar{\varepsilon}} \mu_{j}^{s-\sigma} .
$$

- si $s \leq \sigma-\kappa$, comme $\mu \geq 2^{1 / \kappa} \geq 2$ alors $\mu_{j}^{s-\sigma} \leq \mu_{j}^{-\kappa} \leq \frac{1}{2^{j}}$ et

$$
\left\|w_{n}\right\|_{s} \leq \sum_{j=0}^{n-1} \sqrt{\bar{\varepsilon}} \mu_{j}^{s-\sigma} \leq \sqrt{\bar{\varepsilon}} \sum_{j=0}^{n-1} \frac{1}{2^{j}} \leq 2 \sqrt{\bar{\varepsilon}}
$$

- si $s \geq \sigma+\kappa:$ on a

$$
\left\|w_{n}\right\|_{s} \leq \sqrt{\bar{\varepsilon}} \mu^{s-\sigma}+\sqrt{\bar{\varepsilon}} \frac{\left(\mu^{n(s-\sigma)}-\mu^{s-\sigma}\right)}{\mu^{s-\sigma}-1} .
$$

Or $\mu \geq 2^{1 / \kappa}$ donc $\mu^{s-\sigma} \geq \mu^{\kappa} \geq 2$ et on obtient

$$
\left\|w_{n}\right\|_{s} \leq \sqrt{\bar{\varepsilon}} \mu_{n}^{s-\sigma} .
$$

c) $\left(\right.$ II.65) ${ }_{n}$ : D'après (II.13), (II.15), (II.53), (II.64) ${ }_{n}$ et $5+\left[n_{*}\right] \leq \sigma-\kappa$ on a

$$
\left|\tilde{w}_{n}\right|_{4, \kappa} \leq \beta\left\|\tilde{w}_{n}\right\|_{4+n_{*}} \leq \beta^{2}\left\|w_{n}\right\|_{4+n_{*}} \leq 2 \beta^{2} \sqrt{\bar{\varepsilon}} \leq 1 .
$$

d) (II.66) $)_{n}$ : si $s \leq \sigma+\kappa$, (II.17) et (II.64) $)_{n}$ impliquent:

$$
\begin{aligned}
\left\|w_{n}-\tilde{w}_{n}\right\|_{s} & \leq \beta \mu_{n}^{s-[\sigma+\kappa]-1}\left\|w_{n}\right\|_{[\sigma+\kappa]+1} \\
& \leq \beta \mu_{n}^{[\sigma+\kappa]-1} \sqrt{\bar{\varepsilon}} \mu_{n}^{s-[\sigma+\kappa]+1-\sigma} \leq \beta \sqrt{\bar{\varepsilon}} \mu_{n}^{s-\sigma} .
\end{aligned}
$$

- si $s>\sigma+\kappa$ : (II.15), (II.64) $)_{n}$ et $\beta \geq 1$ donnent

$$
\left\|w_{n}-\tilde{w}_{n}\right\|_{s} \leq 2 \beta\left\|w_{n}\right\|_{s} \leq 2 \beta \sqrt{\bar{\varepsilon}} \mu_{n}^{s-\sigma} .
$$

e) $(\text { II.67) })_{n}$ : D'après (ㅍ․61) on a:

$$
r_{n}=[\underbrace{\left.L_{G}\left(w_{n-1}\right)-L_{G}\left(\tilde{w}_{n-1}\right)\right] u_{n}}_{(1)}-\underbrace{\theta_{n-1} \Delta u_{n}}_{(2)}+\underbrace{Q_{n}}_{(3)} .
$$

Etudions d'abord (1). Dans le cas $n=1,(1)=0$.

- Cas $n \geq 2$ :

$$
(1)=\int_{0}^{1} \frac{d}{d t}\left[L_{G}\left(\tilde{w}_{n-1}+t\left(w_{n-1}-\tilde{w}_{n-1}\right)\right) u_{n}\right] d t .
$$

D'après (II.13), $(\text { II.66) })_{n-1}$ on a

$$
\left|w_{n-1}-\tilde{w}_{n-1}\right|_{2} \leq \beta\left\|w_{n-1}-\widetilde{w}_{n-1}\right\|_{2+n_{*}} \leq 2 \beta^{2} \sqrt{\bar{\varepsilon}} \mu_{n-1}^{3+\left[n_{*}\right]-\sigma}
$$


$2 \beta^{2} \sqrt{\bar{\varepsilon}} \leq 1$ et $3+\left[n_{*}\right] \leq 4+2 n_{*} \leq \sigma$ impliquent alors $\left|w_{n-1}-\tilde{w}_{n-1}\right|_{2} \leq 1$. De même (II.13), (II.53) et (II.63) ${ }_{n}$ donnent

$$
\left|u_{n}\right|_{2} \leq \beta\left\|u_{n}\right\|_{2+n_{*}} \leq \sqrt{\bar{\varepsilon}} \beta \mu_{n-1}^{3+\left[n_{*}\right]-\sigma} \leq 1
$$

et (II.65) $)_{n-1},\left|\tilde{w}_{n-1}\right|_{2} \leq 1$.

On peut donc appliquer la Proposition II.5 et on obtient

$$
\begin{aligned}
\|(1)\|_{s} \leq \bar{\varepsilon} K_{0}\left[\left(\|\varphi\|_{s+2}+\right.\right. & \left.\left\|\tilde{w}_{n-1}\right\|_{s+2}+\left\|w_{n-1}\right\|_{s+2}+1\right)\left|w_{n-1}-\tilde{w}_{n-1}\right|_{2}\left|u_{n}\right|_{2} \\
+\left(\|\varphi\|_{2+n}\right. & \left.+\left\|\tilde{w}_{n-1}\right\|_{2+n_{*}}+\left\|w_{n-1}\right\|_{2+n_{*}}+1\right) \\
& \times\left(\left.\left|w_{n-1}-\tilde{w}_{n-1}\right|\right|_{2}\left\|u_{n}\right\|_{s+2}+\left\|w_{n-1}-\tilde{w}_{n-1}\right\|_{s+2}\left|u_{n}\right|_{2}\right] .
\end{aligned}
$$

D'après (II.18) et (II.50) on a pour $0 \leq s \leq s_{*}$

$$
\|\varphi\|_{s+2} \leq \beta|\varphi|_{s_{*}+2} \leq \beta \mu \leq \mu^{2} .
$$

D'après (II.14) il suffit de prouver (II.67) $n$ pour $s=0$ et $s=s_{*}-2$.

- $s=0$ : (II.13), (II.14), (II.63) $)_{n},(\text { II.64) })_{n-1}$ et (II.66) $)_{n-1}$ impliquent

$$
\begin{aligned}
\|(1)\|_{0} \leq \bar{\varepsilon} K_{0}[ & \left(\mu^{2}+2 \beta \sqrt{\bar{\varepsilon}}+2 \sqrt{\bar{\varepsilon}}+1\right) 2 \beta^{5} \bar{\varepsilon} \mu_{n-1}^{4+2 n_{*}-2 \sigma} \\
& \left.+\left(\mu^{2}+2 \beta \sqrt{\bar{\varepsilon}}+2 \sqrt{\bar{\varepsilon}}+1\right) 4 \beta^{3} \bar{\varepsilon} \mu_{n-1}^{4+n_{*}-2 \sigma}\right] .
\end{aligned}
$$

D'apreś (ㅍ.53) et $\sigma \geq 4+2 n_{*} \geq 4+n_{*}$ on obtient

$$
\|(1)\|_{0} \leq \bar{\varepsilon} K_{0} \mu_{n-1}^{-\sigma} \text {. }
$$

- $s=s_{*}-2$ : D'après (II.53) et $s_{*} \geq \sigma+\kappa$ on obtient comme dans le cas précédent:

$$
\|(1)\|_{s_{*}-2} \leq \bar{\varepsilon} K_{0} \mu_{n-1}^{s_{*}-2-\sigma} .
$$

En utilisant (II.14) on obtient pour $0 \leq s \leq s_{*}-2$

$$
\|(1)\|_{s} \leq K_{0} \beta \mu_{n-1}^{s-\sigma} \text {. }
$$

D'autre part d'après $(\text { II.63 })_{n}$ et (II.69) $)_{n-1}$ on a

$$
\|(2)\|_{s} \leq \theta_{n-1}\left\|u_{n}\right\|_{s+2} .
$$

- si $n=1$ : (II.53), (II.54), (II.58) et (II.63)

$$
\|(2)\|_{s} \leq|G(0)|_{0}\left\|u_{1}\right\|_{s+2} \leq \bar{\varepsilon} \sqrt{\bar{\varepsilon}} \mu^{s+2-\sigma} \leq \bar{\varepsilon} \mu^{s-\sigma} .
$$

- si $n \geq 2$ : (II.63) $)_{n}$ et (II.69) $)_{n-1}$ impliquent

$$
\|(2)\|_{s} \leq a_{3} \bar{\varepsilon} \mu_{n-1}^{-2} \mu_{n-1}^{s+2-\sigma} \leq a_{3} \bar{\varepsilon} \mu_{n-1}^{s-\sigma} .
$$

Enfin d'après (II.62):

$$
\begin{aligned}
(3)=Q_{n} & =G\left(w_{n-1}+u_{n}\right)-G\left(w_{n-1}\right)-L_{G}\left(w_{n-1}\right) u_{n} \\
& =\int_{0}^{1}\left(\int_{0}^{t} \frac{d}{d \tau}\left[L_{G}\left(w_{n-1}+\tau u_{n}\right) u_{n}\right] d \tau\right) d t .
\end{aligned}
$$

D'après (II.13), (II.53) et (II.64) $)_{n-1}$ on a

$$
\left|w_{n-1}\right|_{2} \leq\left\|w_{n-1}\right\|_{2+n_{*}} \leq 2 \beta \sqrt{\bar{\varepsilon}} \leq 1 .
$$

Comme on a aussi déjà montré que $\left|u_{n}\right|_{2} \leq 1$, on peut appliquer la Proposition 【.5. et on obtient

$$
\begin{aligned}
\|(3)\|_{s} \leq & \bar{\varepsilon} K_{0}\left[\left(\|\varphi\|_{s+2}+\left\|u_{n}\right\|_{s+2}+\left\|w_{n-1}\right\|_{s+2}+1\right)\left|u_{n}\right|_{2}^{2}\right. \\
& \left.+2\left|u_{n}\right|_{2}\left\|u_{n}\right\|_{s+2}\left(\|\varphi\|_{2+n_{*}}+\left\|u_{n}\right\|_{2+n_{*}}+\left\|w_{n-1}\right\|_{2+n_{*}}+1\right)\right] .
\end{aligned}
$$


(II.13), (II.14), (II.63) $)_{n}$ et (II.64) $)_{n-1}$ impliquent

- si $s=0$

$\|(3)\|_{0} \leq \bar{\varepsilon} K_{0}\left[\left(\mu^{2}+\sqrt{\bar{\varepsilon}}\left[\max \left(\mu, \mu_{n-1}\right)\right]^{2-\sigma}+2 \sqrt{\bar{\varepsilon}}+1\right) \beta^{4} \bar{\varepsilon}\left[\max \left(\mu, \mu_{n-1}\right)\right]^{4+2 n_{*}-2 \sigma}\right.$

$\left.+2\left(\mu^{2}+\sqrt{\bar{\varepsilon}} \beta\left[\max \left(\mu, \mu_{n-1}\right)\right]^{2+n_{*}-\sigma}+2 \sqrt{\bar{\varepsilon}}+1\right) \bar{\varepsilon} \beta^{2}\left[\max \left(\mu, \mu_{n-1}\right)\right]^{4+n_{*}-2 \sigma}\right]$.

D'après (II.53) et $\sigma \geq 4+2 n_{*}$ on obtient alors

$$
\|(3)\|_{0} \leq \bar{\varepsilon} K_{0}\left[\max \left(\mu, \mu_{n-1}\right)\right]^{-\sigma} .
$$

- si $s=s_{*}-2$, on obtient aussi comme $\sigma \geq 4+2 n_{*}$

$$
\|(3)\|_{s_{*}-2} \leq \varepsilon K_{0}\left[\max \left(\mu, \mu_{n-1}\right)\right]^{s_{*}-2-\sigma} .
$$

D'après (II.14) on a pour $0 \leq s \leq s_{*}-2$

$$
\|(3)\|_{s} \leq K_{0} \beta \bar{\varepsilon}\left[\max \left(\mu, \mu_{n-1}\right)\right]^{s-\sigma} .
$$

(II.79), (III.80), (II.81) et (II.82) impliquent alors:

$$
\begin{aligned}
\left\|r_{n}\right\|_{s} \leq\left(2 K_{0} \beta+a_{3}\right) \bar{\varepsilon}\left[\max \left(\mu, \mu_{n-1}\right)\right]^{s-\sigma} & \leq 9 K_{0} \mu^{5} \bar{\varepsilon}\left[\max \left(\mu, \mu_{n-1}\right)\right]^{s-\sigma} \\
& =a_{1} \bar{\varepsilon}\left[\max \left(\mu, \mu_{n-1}\right)\right]^{s-\sigma} .
\end{aligned}
$$

f) (II.68) $)_{n}$ : D'après (II.68) et (II.69) on a:

$$
\begin{aligned}
f_{n} & =S_{n-1} R_{n-1}-S_{n} R_{n}+\left(S_{n-1}-S_{n}\right) G(0) \\
& =\underbrace{\left(S_{n-1} R_{n-1}-S_{n} R_{n-1}\right)}_{(1)}-\underbrace{S_{n} r_{n}}_{(2)}+\underbrace{\left(S_{n-1}-S_{n}\right) G(0)}_{(3)} .
\end{aligned}
$$

- Cas $s=0$ : (II.17), (II.69) et (II.76) $)_{n}$ impliquent

$$
\begin{aligned}
\|(1)\|_{0} \leq & \left\|\left(I-S_{n-1}\right) R_{n-1}\right\|_{0}+\left\|\left(I-S_{n}\right) R_{n-1}\right\|_{0} \\
\leq & \beta\left\|R_{n-1}\right\|_{s_{*}-2} \mu_{n-1}^{2-s_{*}}+\beta \mu_{n}^{2-s_{*}}\left\|R_{n-1}\right\|_{s_{*}-2} \\
\leq & \beta a_{1} \bar{\varepsilon} \mu_{n-1}^{2-s_{*}}\left(\mu^{s_{*}-2-\sigma}+\sum_{j=2}^{n-1} \mu_{j-1}^{s_{*}-2-\sigma}\right) \\
& +\beta a_{1} \bar{\varepsilon} \mu_{n}^{2-s_{*}}\left(\mu^{s_{*}-2-\sigma}+\sum_{j=2}^{n-1} \mu_{j-1}^{s_{*}-2-\sigma}\right) .
\end{aligned}
$$

Or $s_{*}-2>\sigma$ et $\beta \leq \mu$ donc

$$
\begin{aligned}
\|(1)\|_{0} & \leq \beta a_{1} \bar{\varepsilon}\left(\mu_{n-1}^{2-s_{*}} \mu_{n-1}^{s_{*}-2-\sigma}+\mu_{n}^{2-s_{*}} \mu_{n}^{s_{*}-2-\sigma}\right) \\
& \leq 2 a_{1} \beta \mu^{s_{*}-2} \bar{\varepsilon} \mu_{n}^{-\sigma} \leq 2 a_{1} \mu^{s_{*}-1} \bar{\varepsilon} \mu_{n}^{-\sigma} .
\end{aligned}
$$

D'autre part d'après (II.15), (II.67) $)_{n}, \sigma<s_{*}-2$ et $\beta \leq \mu$ on a:

$$
\|(2)\|_{0} \leq \beta\left\|r_{n}\right\|_{0} \leq \beta \bar{\varepsilon} a_{1}\left[\max \left(\mu, \mu_{n-1}\right)\right]^{-\sigma} \leq\left(\beta a_{1} \mu^{\sigma}\right) \bar{\varepsilon} \mu_{n}^{-\sigma} \leq a_{1} \mu^{s_{*}-1} \bar{\varepsilon} \mu_{n}^{-\sigma} .
$$

Ensuite (III.17), (II.18), (II.54) et $\sigma<s_{*}-2$ donnent:

$$
\begin{aligned}
\|(3)\|_{0} & \leq\left\|\left(I-S_{n-1}\right) G(0)\right\|_{0}+\left\|\left(I-S_{n}\right) G(0)\right\|_{0} \\
& \leq \beta \mu_{n-1}^{-\sigma}\|G(0)\|_{\sigma}+\beta \mu_{n}^{-\sigma}\|G(0)\|_{\sigma} \\
& \leq \beta^{2} \mu_{n-1}^{-\sigma}|G(0)|_{s_{*}}+\beta^{2} \mu_{n}^{-\sigma}|G(0)|_{s_{*}} \\
& \leq \beta^{2} \bar{\varepsilon} \mu_{n}^{-\sigma}\left(\mu^{\sigma}+1\right) \leq 2 \mu^{s_{*}} \bar{\varepsilon} \mu_{n}^{-\sigma} .
\end{aligned}
$$


On obtient finalement

$$
\left\|f_{n}\right\|_{0} \leq\left(2+3 a_{1}\right) \mu^{s_{*}} \bar{\varepsilon} \mu_{n}^{-\sigma} .
$$

- Cas $s=s_{*}$ : D'après (II.16), (II.60) et (II.67) $), 1 \leq j \leq n$ et $\sigma<s_{*}-2$ on a:

$$
\begin{aligned}
\|(1)+(2)\|_{s_{*}} \leq & \left\|S_{n-1} R_{n-1}\right\|_{s_{*}}+\left\|S_{n} R_{n}\right\|_{s_{*}} \\
\leq & \beta \mu_{n-1}^{2}\left\|R_{n-1}\right\|_{s_{*}-2}+\beta \mu_{n}^{2}\left\|R_{n}\right\|_{s_{*}-2} \\
\leq & \beta \mu_{n-1}^{2} a_{1} \bar{\varepsilon}\left(\mu^{s_{*}-2-\sigma}+\sum_{j=2}^{n-1} \mu_{j-1}^{s_{*}-2-\sigma}\right) \\
& +\beta \mu_{n}^{2} a_{1} \bar{\varepsilon}\left(\mu^{s_{*}-2-\sigma}+\sum_{j=2}^{n} \mu_{j-1}^{s_{*}-2-\sigma}\right) \\
\leq & \beta a_{1} \bar{\varepsilon}\left(\mu_{n-1}^{2} \mu_{n-1}^{s_{*}-2-\sigma}+\mu_{n}^{2} \mu_{n}^{s_{*}-2-\sigma}\right) \\
\leq & 2 \beta a_{1} \bar{\varepsilon} \mu_{n}^{s_{*}-\sigma} \leq 2 \mu a_{1} \bar{\varepsilon} \mu_{n}^{s_{*}-\sigma} .
\end{aligned}
$$

D'autre part (II.16), (II.18), (II.54) et $\beta \leq \mu$ impliquent:

$$
\begin{aligned}
\|(3)\|_{s_{*}} & \leq\left\|S_{n} G(0)\right\|_{s_{*}}+\left\|S_{n-1} G(0)\right\|_{s_{*}} \\
& \leq \beta \mu_{n}^{s_{*}-\sigma}\|G(0)\|_{\sigma}+\beta \mu_{n-1}^{s_{*}-\sigma}\|G(0)\|_{\sigma} \\
& \leq 2 \beta^{2} \bar{\varepsilon} \mu_{n}^{s_{*}-\sigma} \leq 2 \mu^{2} \bar{\varepsilon} \mu_{n}^{s_{*}-\sigma}
\end{aligned}
$$

d'où

$$
\left\|f_{n}\right\|_{s_{*}} \leq 2 \mu\left(a_{1}+\mu\right) \bar{\varepsilon} \mu_{n}^{s_{*}-\sigma} .
$$

Finalement d'après (III.14), (II.84), (II.85) et $\mu \leq a_{1}$ on obtient

$$
\left\|f_{n}\right\|_{s} \leq 5 \beta a_{1} \mu^{s_{*}} \bar{\varepsilon} \mu_{n}^{s-\sigma} \leq 5 a_{1} \mu^{s_{*}+1} \bar{\varepsilon} \mu_{n}^{s-\sigma}=a_{2} \bar{\varepsilon} \mu_{n}^{s-\sigma} .
$$

g) (II.69) ${ }_{n}$ : D'après (II.58) on a

$$
\theta_{n}=\left|G\left(\tilde{w}_{n}\right)\right|_{0} \leq\left|G\left(w_{n}\right)-G\left(\tilde{w}_{n}\right)\right|_{0}+\left|G\left(w_{n}\right)\right|_{0} .
$$

D'autre part (II.56) $)_{n}$ à (III.62) impliquent:

$$
G\left(w_{n}\right)=\left(I-S_{n-1}\right) R_{n-1}+\left(I-S_{n-1}\right) G(0)+r_{n},
$$

donc

$$
\theta_{n} \leq \underbrace{\left|G\left(w_{n}\right)-G\left(\widetilde{w}_{n}\right)\right|_{0}}_{(1)}+\underbrace{\left|\left(I-S_{n-1}\right) R_{n-1}\right|_{0}}_{(2)}+\underbrace{\left|\left(I-S_{n-1}\right) G(0)\right|_{0}}_{(3)}+\underbrace{\left|r_{n}\right|_{0}}_{(4)} .
$$

D'après un calcul précédent on a $\left|w_{n}\right|_{2} \leq 1$ et $\left|\tilde{w}_{n}\right|_{2} \leq 1$, on peut donc appliquer la Proposition 11.5 et on obtient:

$$
(1) \leq \beta K_{0}\left\|w_{n}-\tilde{w}_{n}\right\|_{2+n_{*}}\left(\|\varphi\|_{2+n_{*}}+\left\|w_{1}\right\|_{2+n_{*}}+\left\|\tilde{w}_{n}\right\|_{2+n_{*}}\right) .
$$

(II.14), (II.64) $)_{n},(\text { II. } 6)_{n}$ et $3+\left[n_{*}\right] \leq 4+2 n_{*}-\kappa \leq \sigma-\kappa$ donnent

$$
\text { (1) } \leq 2 \beta^{3} K_{0} \sqrt{\bar{\varepsilon}} \mu_{n}^{2+n_{*}-\sigma}\left(\mu^{2}+2 \sqrt{\bar{\varepsilon}}+2 \beta \sqrt{\bar{\varepsilon}}\right) .
$$

Or $\bar{\varepsilon} \leq \frac{1}{\left(6 \beta^{2}\right)^{2}}, \beta \leq \mu$ et $4+n_{*}-\sigma \leq 4+2 n_{*}-\sigma \leq 0$ donc

$$
\text { (1) } \leq 4 \mu^{5} K_{0} \sqrt{\bar{\varepsilon}} \mu_{n}^{-2} \text {. }
$$


Ensuite (II.13), (II.17) et (II.67) $] 1 \leq j \leq n$ impliquent pour $n \geq 2$

$$
\begin{aligned}
(2) & \leq \beta\left\|\left(I-S_{n-1}\right) R_{n-1}\right\|_{n_{*}} \\
& \leq \beta^{2} \mu_{n-1}^{n_{*}-s_{*}+2} a_{1} \bar{\varepsilon}\left(\mu^{s_{*}-2-\sigma}+\sum_{j=2}^{n-1} \mu_{j-1}^{s_{*}-2-\sigma}\right) \\
& \leq \beta^{2} a_{1} \bar{\varepsilon} \mu_{n-1}^{n_{*}-\sigma}
\end{aligned}
$$

or $\beta \leq \mu, n_{*}-\sigma \leq-2$, et $\mu^{4} a_{1} \sqrt{\bar{\varepsilon}} \leq a_{2} \sqrt{\bar{\varepsilon}} \leq 1$, donc

$$
(2) \leq \mu^{4} a_{1} \bar{\varepsilon} \mu_{n}^{-2} \leq \sqrt{\bar{\varepsilon}} \mu_{n}^{-2},
$$

et pour $n=1,(2)=0$.

D'autre part (ㅍ.13), (II.17), (II.53), (I.54) et $\beta \leq \mu$ donnent:

$$
\begin{aligned}
(3) \leq \beta\left\|\left(I-S_{n-1}\right) G(0)\right\|_{n_{*}} & \leq \beta^{2} \mu_{n-1}^{n_{*}-s_{*}}\|G(0)\|_{s_{*}} \\
& \leq \beta^{3} \mu_{n-1}^{-2} \bar{\varepsilon} \leq \beta^{3} \mu^{2} \bar{\varepsilon} \mu_{n}^{-2} \leq \sqrt{\bar{\varepsilon}} \mu_{n}^{-2} .
\end{aligned}
$$

Finalement (II.13) et (II.67) $n$ impliquent

$$
\text { (4) } \leq \beta\left\|r_{n}\right\|_{n_{*}} \leq \beta^{2} a_{1} \bar{\varepsilon}\left[\max \left(\mu, \mu_{n-1}\right)\right]^{n_{*}-\sigma} .
$$

Or $\beta \leq \mu, n_{*}-\sigma \leq-4-n_{*} \leq-2$ et $a_{1} \mu^{4} \sqrt{\bar{\varepsilon}} \leq a_{2} \sqrt{\bar{\varepsilon}} \leq 1 \mathrm{donc}$

$$
\text { (4) } \leq \mu^{2} a_{1} \bar{\varepsilon}\left[\max \left(\mu, \mu_{n-1}\right)\right]^{-2} \leq \mu^{4} a_{1} \bar{\varepsilon} \mu_{n}^{-2} \leq \sqrt{\bar{\varepsilon}} \mu_{n}^{-2} .
$$

(II.51), (II.53), (II.86), (II.87), (II.88) et (II.89) impliquent alors:

$$
\theta_{n} \leq 7 K_{0} \mu^{5} \sqrt{\bar{\varepsilon}} \mu_{n}^{-2}=a_{3} \sqrt{\bar{\varepsilon}} \mu_{n}^{-2} \leq a_{2} \sqrt{\bar{\varepsilon}} \leq 1 .
$$

h) $(\text { II. } 70)_{n}:$ On a

$$
A_{n}(2) \leq \max \left(1, \max _{1 \leq i, j \leq n}\left|\frac{\partial F}{\partial u_{i j}}\left(\varphi_{p q}+\bar{\varepsilon}\left(\tilde{w}_{n}\right)_{p q}\right)\right|_{2}+\theta_{n}\right) .
$$

D'après (II.20), (II.48), (II.65) ${ }_{n}$ et (II.69) $)_{n}$ on obtient $A_{n}(2) \leq M_{0}$.

Maintenant on va montrer à l'aide de la Proposition $\amalg .12$ la convergence de la suite $\left(w_{n}\right)$.

Prenons $\sigma=s_{*}-2-\kappa$ et $s=\sigma-\kappa$. On a d'après [II.55), (II.63) $)_{j}$ et pour tous $i, k \in \mathbb{N}^{*}, i>k$

$$
\left\|w_{i}-w_{k}\right\|_{s} \leq \sum_{j=k+1}^{i}\left\|u_{j}\right\|_{s} \leq \beta \sqrt{\bar{\varepsilon}} \sum_{j=k+1}^{i} \mu_{j-1}^{-\kappa}=\beta \sqrt{\bar{\varepsilon}} \sum_{j=k+1}^{i}\left[\mu^{-\kappa}\right]^{j-1}
$$

or $\mu \geq 2, \kappa>0$ donc $\left\|w_{i}-w_{k}\right\|_{s}$ tend vers zéro lorsque $i$ et $j$ tendent vers l'infini. Il existe donc une fonction $w \in H^{s_{*}-2-2 \kappa}(\Omega)$ telle que $\left(w_{n}\right)$ converge vers $w$. D'après l'injection de Sobolev $w \in C^{s_{*}-2-\frac{n}{2}-2 \kappa}(\Omega) \subset C^{\left[s_{*}-3-\frac{n}{2}\right]}(\Omega)$. D'autre part

$$
G\left(w_{n}\right)=\left(I-S_{n-1}\right) R_{n-1}+\left(I-S_{n-1}\right) G(0)+r_{n} .
$$

On a d'après (III.14) et (II.67) $)_{n}$ pour $n \geq 2$

$$
\left\|r_{n}\right\|_{s_{*}-2-2 \kappa} \leq a_{1} \beta \bar{\varepsilon} \mu_{n-1}^{s_{*}-2-2 \kappa-\sigma}=a_{1} \beta \bar{\varepsilon} \mu_{n-1}^{-\kappa} .
$$


(II.17), (II.18) et (II.54) impliquent:

$$
\left\|\left(I-S_{n-1}\right) G(0)\right\|_{s_{*}-2-2 \kappa} \leq \beta \mu_{n-1}^{-2-2 \kappa}\|G(0)\|_{s_{*}} \leq \beta^{2} \mu_{n-1}^{-2-2 \kappa} \bar{\varepsilon} .
$$

Enfin (II.17) et (II.67) $)_{j}, 1 \leq j \leq n$ donnent:

$$
\begin{aligned}
\|(I- & \left.S_{n-1}\right) R_{n-1} \|_{s_{*}-2-2 \kappa} \\
& \leq \beta \mu_{n-1}^{-2 \kappa}\left\|R_{n-1}\right\|_{s_{*}-2} \leq \beta \mu_{n-1}^{-2} \sum_{j=1}^{n-1}\left\|r_{j}\right\|_{s_{*}-2} \\
& \leq \beta \mu_{n-1}^{-2 \kappa} \bar{\varepsilon} a_{1}\left(\mu^{s_{*}-2-\sigma}+\sum_{j=2}^{n-1} \mu_{j-1}^{s_{*}-2-\sigma}\right) \\
& \leq \bar{\varepsilon} \beta a_{1} \mu_{n-1}^{-2 \kappa} \mu_{n-1}^{s_{*}-2-\sigma} \leq \beta a_{1} \bar{\varepsilon} \mu_{n-1}^{-\kappa} .
\end{aligned}
$$

(III.90) à (II.92) montrent que $G\left(w_{n}\right)$ tend vers zéro dans $H^{s_{*}-2-2 \kappa}(\Omega)$ lorsque $n$ tend vers l'infini donc $G(w)=0$ car $H^{s_{*}-2-2 \kappa}(\Omega) \subset C^{2}(\Omega)$. De plus $\left.w_{n}\right|_{\partial \Omega}=0$, d'où $\left.w\right|_{\partial \Omega}=0$. On obtient alors la solution du Théorème $₫$ en posant $u=\varphi+\bar{\varepsilon} w$. D'après le Lemme II.2 et comme $f$ est positive, cette solution est convexe; l'unicité découle du principe du maximum (cf. [C.N.S.2]).

\section{BIBLIOGRAPHIE}

[A.G] S. Alinhac, P. Gérard: Opérateurs pseudo-différentiels et théorème de Nash-Moser, InterEditions et Editions du CNRS, 1991. |MR 93g:35001]

[A] K. Amano: The Dirichlet problem for degenerate elliptic 2-dimensional Monge-Ampère equation, Bull. Austral. Math. Soc. 37 (1988), 389-410. MR 89h:35115a

[C.N.S.1] L. Caffarelli, L. Nirenberg, J. Spruck: The Dirichlet problem for nonlinear second-order elliptic equations. I. Monge-Ampère equation, Comm. Pure Appl. Math. 37 (1984), 369402. MR 87f:35096

[C.N.S.2] L. Caffarelli, L. Nirenberg, J. Spruck: The Dirichlet problem for nonlinear second-order elliptic equations. II. Complex Monge-Ampère and uniformly elliptic equations, Comm. Pure Appl. Math. 38 (1985), 209-252. MR 87f:35097

[C.N.S.3] L. Caffarelli, L. Nirenberg, J. Spruck: The Dirichlet problem for the degenerate MongeAmpère equation, Rev. Mat. Iberoamericana 2 (1986), 19-27. MR 87m:35103

[E] L. Evans: Classical solutions of fully nonlinear, convex, second-order elliptic equations, Comm. Pure Appl. Math. 25 (1982), 333-363. MR 83g:35038

[G.T] D. Gilbarg, N. S. Trudinger: Elliptic partial differential equations of second order, 2nd ed., Springer-Verlag, Berlin and New York, 1983. MR 86c:35035

[H.Z] J. Hong, C. Zuily: Existence of $C^{\infty}$ local solutions for the Monge-Ampère equation, Invent. Math. 89 (1987), 645-661. MR 88j:35056

[Hör] L. Hörmander: On the Nash-Moser implicit function theorem, Acad. Sci. Fenn. Ser. A I Math. 10 (1985), 255-259. MR 87a:58025]

[K] N. V. Krylov: Boundedly nonhomogeneous elliptic and parabolic equations in a domain, Math. USSR Izv. 22 (1984), 67-97.

[L] C. S. Llin, The local isometric embedding in $\mathbb{R}^{3}$ of two dimensional Riemannian manifolds with nonnegative curvature, J. Differential Geom. 21 (1985), 213-230. MR 87m:53073

[O.R] O. A. Oleinik, E. V. Radkevich: Second order equations with non negative characteristic form, Plenum Press, New York, 1973. MR 56:16112

[T] N. S. Trudinger: Fully nonlinear, uniformly elliptic equations under natural structure conditions, Trans. Amer. Math. Soc. 278 (1983), 751-769. MR 85b:35016 
[U] J. I. E. Urbas: Elliptic equations of Monge-Ampère type, Thesis, Australian National University, 1984.

[Z] C. Zuily: Sur la régularité des solutions non strictement convexes de l'équation de Monge-Ampère réelle, Ann. Scuola Norm. Sup. Pisa Cl. Sci. (4) 15 (1988), 529-554. MR 91e:35095

Université de Paris-Sud, Département de Mathématiques, Bât. 425, 91405 Orsay, Cedex, France

Current address: Département de Mathématiques, Faculté des Sciences de Tunis, Campus Universitaire le Belvedere, 1060 Tunis, Tunisie

E-mail address: sami.baraket@fst.rnu.tn 